\title{
SINGULARITIES OF CONVEX HULLS AS FRONTS OF LEGENDRE VARIETIES
}

\author{
ILIA A. BOGAEVSKI \\ Independent University of Moscow \\ Bol'shoŭ Vlas'evskiǔ per. 11, Moscow 121002, Russia \\ The University of Aizu \\ Aizu-Wakamatsu City, Fukushima 965-8580, Japan \\ E-mail: bogaevsk@msfu.msk.ru,bogaevsk@u-aizu.ac.jp
}

\begin{abstract}
We describe singularities of the convex hull of a generic compact smooth hypersurface in four-dimensional affine space up to diffeomorphism. It turns out that the boundary of the convex hull is the front of a Legendre variety. Its singularities are classified up to contact diffeomorphism.
\end{abstract}

1. Introduction. The convex hull of a compact subset of an affine space is the intersection of all closed half-spaces which contain the subset. The boundary of the convex hull of a compact smooth hypersurface can have singularities. For example, the singularities of the boundary of the convex hull of a generic closed plane curve are discontinuities of the second derivative (Fig. 1).

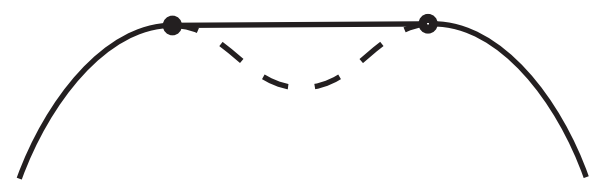

Figure 1. Singularities of the convex hull of a generic plane curve

In the present paper we describe, up to diffeomorphism, singularities of the convex hulls of generic compact $C^{\infty}$-smooth hypersurfaces without boundary embedded into four-dimensional affine space. A singularity of a convex hull is its germ at a singular point of the boundary. As usual, generic hypersurfaces are embeddings which form an open everywhere dense set in the $C^{\infty}$-space of all embeddings considered. In other words, we

1991 Mathematics Subject Classification: Primary 58C27; Secondary 52A20.

Supported by RFBR-96-01-01104, RFBR-96-15-96043, and INTAS-96-0713.

The paper is in final form and no version of it will be published elsewhere. 
are interested only in singularities which are not removed by any $C^{\infty}$-small perturbation of the original hypersurface.

It turns out there are only two new singularities (in comparison with dimension three) which appear at isolated points of the boundary of the convex hull and are not removed by small perturbation of the original hypersurface. The first singularity does not contain functional moduli, but has at least nine numerical ones. A normal form which does not contain moduli at all is found for the second singularity.

Moreover, we show that the boundary of the convex hull is the front of a Legendre variety and we find normal forms of its germs with respect to contact diffeomorphisms. All singularities of the Legendre variety prove to be stable and simple in contrast to the singularities of the convex hull itself.

A tangent hyperplane is called a support hyperplane if the hypersurface lies entirely in one of the two closed half-spaces defined by the hyperplane. A support hyperplane is called non-singular if it has only one common point with the hypersurface and this point is a point of non-degenerate tangency. All the remaining support planes are called singular. For example, the singular support hyperplanes to a generic plane curve are straight lines of double non-degenerate tangency. Singularities of the convex hull of a compact hypersurface can appear only in its singular support hyperplanes.

1.1. Three-dimensional space. The convex hull of a generic compact surface in threedimensional space can have only two kinds of singularities which we call simplest and angle singularities. The normal form of the angle singularity contains a numerical modulus (continuous invariant) with respect to diffeomorphisms. These singularities shown in Fig. 2 are found in [7]. The results of this paper are the following.

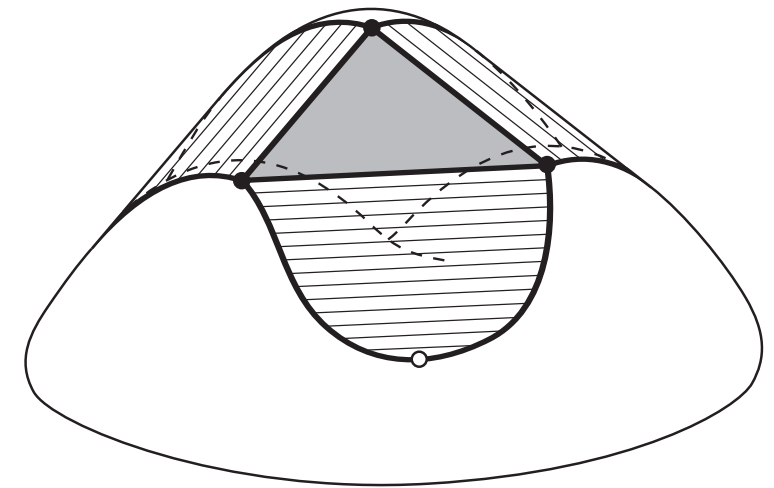

Figure 2. Singularities of the convex hull of a generic surface

A typical singular support hyperplane to a generic surface in three-dimensional space has two (and only two) points of non-degenerate tangency with the surface $\left(2 A_{1}\right.$-plane). Isolated singular support hyperplanes can have either three points of non-degenerate tangency with the surface which form a triangle or one point of degenerate tangency $A_{3}$. Such hyperplanes are called $3 A_{1}$ - and $A_{3}$-planes respectively.

The segment between the points of tangency of a surface and its support $2 A_{1}$-plane is called a support segment. It lies entirely in the boundary of the convex hull of the 
original surface. The boundary is smooth at the interior points of the support segment. In neighborhoods of the endpoints of the support segment the boundary is diffeomorphic to the product of a line and a curve with the singularity shown in Fig. 1. Such singularities of a convex hull are called simplest and denoted by $\mathcal{R}_{1}$.

The triangle with vertices at the points of tangency of a surface and its support $3 A_{1}$-plane is called a support triangle. In its neighborhood the boundary of the convex hull of the surface consists of the support triangle itself, three smooth surfaces webbed from support segments and adjoining the sides of the support triangle, and three parts of the original surface adjoining the vertices of the support triangle. The convex hull has the simplest singularities at the interior points of the sides of the support triangle. To describe singularities at its vertices let us define the under-graph as the set of points lying under the graph of a given function. It turns out that in a neighborhood of each vertex of the support triangle the convex hull is diffeomorphic to the under-graph of the square of the distance to an angle of a value $\beta$ where $0<\beta<\pi$ is a unique modulus in the normal form. Such singularities of a convex hull are called angle singularities. Let us note that we can consider the epigraph of the square of the distance instead of its under-graph they become diffeomorphic after the substitution $\beta \mapsto \pi-\beta$.

Finally, the convex hull of a generic surface has the simplest singularity at any support $A_{3}$-point that is a point of tangency of the surface and its support $A_{3}$-plane. In a neighborhood of such a point the boundary of the convex hull consists of a part of the original surface and a surface webbed from support segments which degenerate into the support $A_{3}$-point.

1.2. Four-dimensional space. Some singularities of the convex hull of a generic hypersurface in four-dimensional space are investigated in [5] and [6]. In [5] normal forms of singularities of convex hulls are found in the case when the support hyperplane is tangent to the original hypersurface in one of the ways described above for three-dimensional space. Thus the simplest and angle singularities appear in four-dimensional space as well.

The boundary of the convex hull is smooth at the interior points of the support segments of the $2 A_{1}$-planes and has the simplest singularities at their endpoints. The support segments themselves lie entirely in the boundary of the convex hull of the original hypersurface.

At the interior points of the support triangles of the $3 A_{1}$-planes the boundary of the convex hull is smooth and has the simplest singularities at the interior points of their sides. The support triangles themselves lie entirely in the boundary of the convex hull of the original hypersurface. In a neighborhood of each of their vertices the convex hull is diffeomorphic to the curvilinear cylinder over the above angle singularity with $\beta(z)=\beta_{0}+z, \beta(z)=\beta_{0}+z^{2}$, or $\beta(z)=\beta_{0}-z^{2}$ where $0<\beta_{0}<\pi$ is a unique modulus in each of the three normal forms and $z$ is a coordinate along the element of the cylinder. Such singularities of a convex hull are called angle singularities and denoted by $\mathcal{R}_{2}^{0}, \mathcal{R}_{2}^{+}$, and $\mathcal{R}_{2}^{-}$respectively.

Finally, the convex hull of a generic hypersurface has the simplest singularity at any support $A_{3}$-point again.

However, among the support hyperplanes of a three-dimensional hypersurface there 
can be new $4 A_{1}$ - and $A_{1} A_{3}$-planes which are not removed by any small perturbation of the hypersurface. Each $4 A_{1}$-plane has four points of non-degenerate tangency with the hypersurface which form a tetrahedron. Each $A_{1} A_{3}$-plane has one point of non-degenerate tangency and one point of tangency $A_{3}$.

The tetrahedron with vertices at the points of tangency of a hypersurface and its support $4 A_{1}$-plane is called a support tetrahedron. In its neighborhood the boundary of the convex hull of the hypersurface consists of the support tetrahedron itself, four smooth strata webbed from support triangles and adjoining the faces of the support tetrahedron, six smooth strata webbed from support segments and adjoining the edges of the support tetrahedron, and four parts of the original hypersurface adjoining the vertices of the support tetrahedron. Thus in this case the singular points of the boundary divide it into $15(1+4+6+4)$ strata as shown in Fig. 3, left.

To imagine a convex hull in four-dimensional space it is convenient to project it affinely into the support hyperplane to the original hypersurface. Then the boundary of the convex hull is locally the graph of a continuously differentiable function (see, e.g., [7]) whose typical singularities are discontinuities of the second derivative. In a neighborhood of a support tetrahedron of a generic three-dimensional hypersurface the points of such discontinuities form 28 smooth strata which are shown in Fig. 3, left.
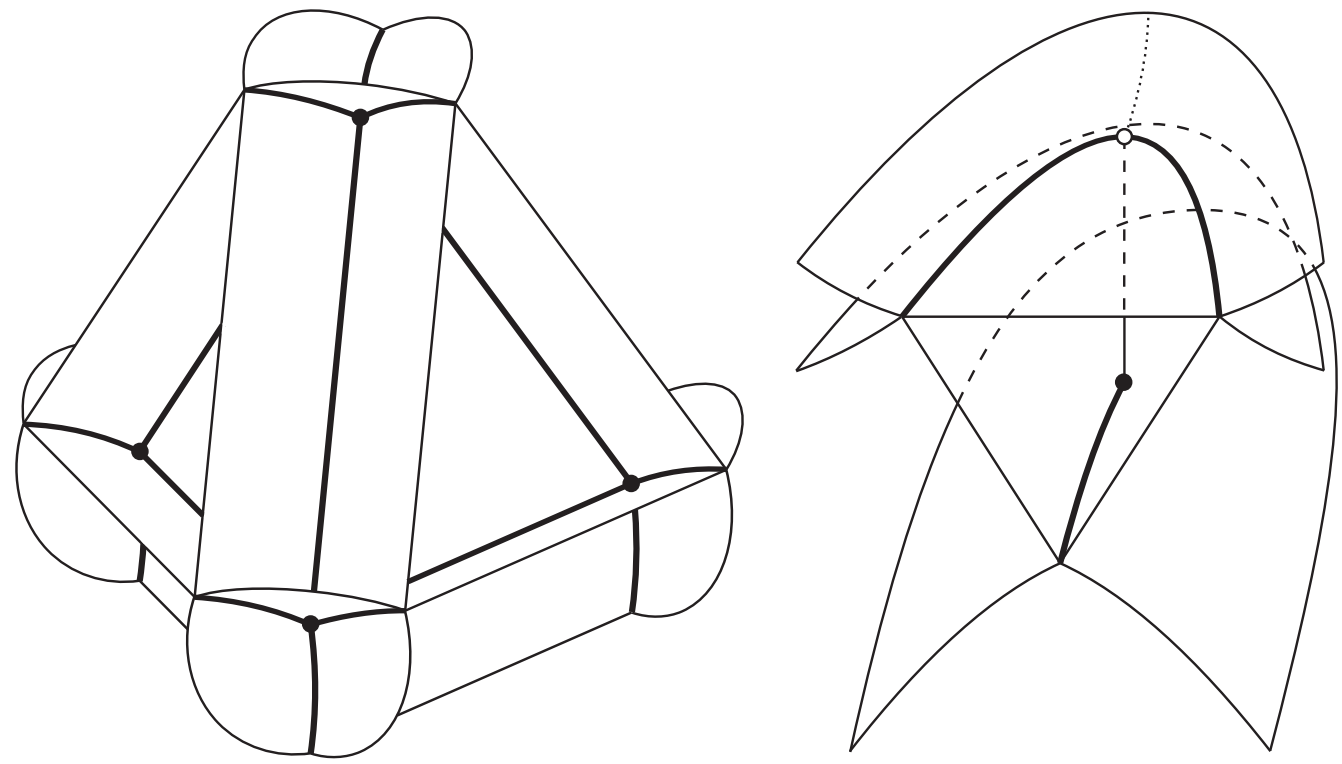

Figure 3. Singularities of the convex hull of a generic three-dimensional hypersurface in a neighborhood of a support tetrahedron and in a neighborhood of a support

$$
A_{1} A_{3} \text {-segment }
$$

According to [5], the convex hull of a generic three-dimensional hypersurface has the simplest singularities at the interior points of the faces of a support tetrahedron and the angle singularities at the interior points of its edges. In the present paper it is proved that the germ of our convex hull at no vertex of a support tetrahedron contains functional 
moduli with respect to diffeomorphisms. This singularity is denoted by $\mathcal{R}_{3}$. Its normal form is not found nor the precise number of numerical moduli. It is only proved certainly not to be less than nine but apparently is much more.

Moreover, we investigate singularities in the only remaining case when a generic hypersurface has a support $A_{1} A_{3}$-plane. The segment between their tangency points is called the support $A_{1} A_{3}$-segment. It lies entirely in the boundary of the convex hull of the original hypersurface. According to our results, the convex hull of a generic three-dimensional hypersurface has the simplest singularities at the interior points of its support $A_{1} A_{3^{-}}$ segment and the angle singularity $\mathcal{R}_{2}^{0}$ at the endpoint $A_{3}$. At the endpoint $A_{1}$ of the support $A_{1} A_{3}$-segment there appears one more singularity of the convex hull. This singularity is denoted by $\mathcal{V}_{3}$, does not contain functional moduli, and is diffeomorphic to its normal form. This normal form is the under-graph of the square of the distance to that component of the complement to the swallowtail which consists of polynomials without real roots.

In a neighborhood of a support $A_{1} A_{3}$-segment the boundary of the convex hull of a generic hypersurface is divided by the singular points into five strata four of which are smooth and the fifth one is non-smooth - this phenomenon has not occurred before. The projection of the singular points of the boundary of the convex hull into the support $A_{1} A_{3^{-}}$ plane is shown in Fig. 3, right. It consists of three smooth surfaces, the cut swallowtail, and half of the Whitney umbrella. The support $A_{1} A_{3}$-segment lies in the Whitney umbrella, its endpoint $A_{3}$ is the starting point of a smooth curve which consists of support $A_{3}$-points and is shown by dots in Fig. 3. It should be noted that in general the normalizing diffeomorphisms preserve neither the support $A_{1} A_{3}$-segment, nor the curve of support $A_{3}$-points.

Inside the cut swallowtail the boundary of the convex hull is the original hypersurface. A smooth stratum webbed from support triangles which degenerate into the support $A_{1} A_{3}$-segment is inside the Whitney umbrella. The stratum between these two is non-smooth and webbed from support segments. One more part of the original hypersurface bounded by two smooth surfaces adjoins the non-singular stratum. The remaining smooth stratum is bounded by two surfaces too and is webbed from support segments degenerating into support $A_{3}$-points.

The union of the cut swallowtail and the half Whitney umbrella whose intersection lines coincide and whose tangent cones are transversal is called a sail-boat in [6]. There it is proved that the projection of the singular points of the boundary of the convex hull into a support $A_{1} A_{3}$-plane to a generic hypersurface is a sail-boat in a neighborhood of the point of tangency $A_{1}$ and all sail-boats are diffeomorphic to each other. According to our results, in this case the convex hull itself is reduced to the above local normal form which does not contain moduli as well.

The following conjecture is formulated in [4]: the singularities of the convex hull of a generic compact hypersurface in four-dimensional affine space do not contain functional moduli. Thus this conjecture is proved in the present paper.

So in a neighborhood of a typical point of the boundary the convex hull of a generic compact three-dimensional hypersurface is diffeomorphic to the closed half-space. The 
points of the boundary where the convex hull has the simplest singularities $\mathcal{R}_{1}$ form a smooth two-dimensional surface. Finally, the angle singularities $\mathcal{R}_{2}^{0}$ appear along smooth curves, the singularities $\mathcal{R}_{2}^{+}, \mathcal{R}_{2}^{-}, \mathcal{R}_{3}$, and $\mathcal{V}_{3}$ appear at isolated points of the boundary of the convex hull.

The singularities $\mathcal{R}_{1}, \mathcal{R}_{2}^{0}, \mathcal{R}_{2}^{ \pm}$, and $\mathcal{V}_{3}$ allow the following uniform representations: the simplest singularity $\mathcal{R}_{1}$ is diffeomorphic to the under-graph of the square of the distance to a three-dimensional half-space, the angle singularity $\mathcal{R}_{2}^{0}$ or $\mathcal{R}_{2}^{ \pm}$is diffeomorphic to the under-graph of the square of the distance to a dihedral angle of variable value $\beta(z)$ where $\beta(z)=\beta_{0}+z$ or $\beta(z)=\beta_{0} \pm z^{2}$ respectively, and the singularity $\mathcal{V}_{3}$ is diffeomorphic to the under-graph of the square of the distance to one of the components of the complement to the swallowtail. For the singularity $\mathcal{R}_{3}$ there is apparently no analogous representation.

1.3. Singularities of Legendre varieties. It turns out that the boundary of the convex hull of a generic compact three-dimensional hypersurface is the front of a Legendre variety which can have only the following standard singularities: $\widetilde{R_{1}}, \widetilde{R_{2}}, \widetilde{R_{3}}$, and $\widetilde{V_{3}}$. These singularities do not have moduli with respect to contact diffeomorphisms. The singularities $\widetilde{R_{1}}$ form smooth two-dimensional surfaces, the singularities $\widetilde{R_{2}}$ appear along smooth curves, and the singularities $\widetilde{R_{3}}$ and $\widetilde{V_{3}}$ appear at isolated points of the Legendre variety. The singularities $\widetilde{R_{1}}$ are projected to the simplest singularities of the convex hull, the singularities $\widetilde{R_{2}}$ are projected to the angle ones, the singularities $\widetilde{R_{3}}$ are projected to the singularities $\mathcal{R}_{3}$, and the singularities $\widetilde{V_{3}}$ are projected to the singularities $\mathcal{V}_{3}$.

The singularities $\widetilde{R_{l}}$ consist of $2^{l}$ smooth strata. The singularity $\widetilde{V_{3}}$ consists of one non-smooth and two smooth strata. If $l=3$, the family

$$
\Phi_{l}(\tau, \lambda)=\frac{1}{2}\left(\tau^{l+1}+\lambda_{1} \tau^{l-1}+\ldots+\lambda_{l-1} \tau+\lambda_{l}\right)^{2}
$$

generates one of the smooth strata of the singularity $\widetilde{V_{3}}$ and the non-smooth one. According to Theorem 8 from [8], all families $\Phi_{l}$ generate Legendre (Lagrangian) varieties $\widetilde{\Phi_{l}}$ whose generic Legendre (Lagrangian) projections are stable in the sense of 3.3 in [2]. Open Whitney umbrellas and open swallowtails possess the same property as well, but our varieties $\widetilde{\Phi_{l}}$ are new if $l \geq 3\left(\widetilde{\Phi_{1}}\right.$ is an intersection of curves and $\widetilde{\Phi_{2}}$ is the open Whitney umbrella).

1.4. Higher dimensions. According to [3], in dimension five or more the convex hull can have functional moduli which are not removed by small perturbation of the original hypersurface. For example, they appear as relations between several numerical moduli (which are already in four-dimensional space) along the lines formed by the vertices of the support tetrahedrons.

1.5. Terminology. The term "smooth" always means "infinitely smooth". The term "generic" is used for smooth mappings and means that the given proposition is only true for some open everywhere dense set in the $C^{\infty}$-space of all mappings considered. The term "typical" is used for points of varieties and means that the given proposition is only true for some open everywhere dense set of points. 
1.6. Organization of the paper. In Section 2 we give the necessary definitions and rigorously formulate the results (Theorems 1 and 2) outlined in Introduction. In Section 3 the proof of these results is divided into two steps which are Theorems 3 and 4 proved in $[1]$.

Theorem 3 from Section 3 states that the boundary of the convex hull of a generic compact three-dimensional hypersurface is the front of a Legendre variety which can have only the standard singularities: $\widetilde{R_{1}}, \widetilde{R_{2}}, \widetilde{R_{3}}$, and $\widetilde{V_{3}}$. Their normal forms with respect to contact diffeomorphisms are described in Section 3.

Theorem 4 from Section 3 contains the classification of germs of a generic Legendre bundle at points of the Legendre variety up to contact diffeomorphisms preserving its local normal forms $\widetilde{R_{1}}, \widetilde{R_{2}}, \widetilde{R_{3}}$, and $\widetilde{V_{3}}$ already found. However, we consider only bundles with the following property: the front of the Legendre variety must be a continuously differentiable manifold. (The boundary of the convex hull of any compact hypersurface is continuously differentiable.)

Acknowledgments. The author is grateful to V. D. Sedykh for statement of the problem and interest in the paper, and to V. V. Goryunov and Yu. G. Prokhorov for numerous useful discussions.

\section{Classification of singularities of convex hulls}

Definition 1. The convex hull of a compact subset of an affine space is the intersection of all closed half-spaces which contain the subset.

Definition 2. A hyperplane is called a support hyperplane to a subset of an affine space if the subset lies entirely in one of the two half-spaces defined by the hyperplane and has at least one common point with the hyperplane.

If the subset is a manifold then so-called $A_{l_{1}} \ldots A_{l_{m}}$-planes are distinguished among its support hyperplanes.

Definition 3. A support hyperplane to a manifold is called an $A_{l_{1}} \ldots A_{l_{m}}$-plane $\left(l_{1}, \ldots, l_{m}\right.$ are positive odd numbers) if:

1) it has $m$ points of tangency $A_{l_{1}}, \ldots, A_{l_{m}}$ with the manifold;

2) these points of tangency are the vertices of an $(m-1)$-dimensional simplex which is called a support simplex of the $A_{l_{1}} \ldots A_{l_{m}}$-plane or a support $A_{l_{1}} \ldots A_{l_{m}}$-simplex.

A support $A_{1}$-plane is called non-singular. The remaining support hyperplanes are called singular.

Remark 1 . If $l_{1}=\ldots=l_{m}=1$ then we write $m A_{1}$ instead of $A_{1} \ldots A_{1}$.

Remark 2. A $k$-dimensional manifold has a point of tangency $A_{l}$ ( $l$ is a positive integer) with a hypersurface if the restriction of some local equation of the hypersurface has the form $\pm \xi_{1}^{l+1} \pm \xi_{2}^{2} \pm \ldots \pm \xi_{k}^{2}$ in suitable local coordinates on the manifold. 
According to [7], if $k=1$ or $n \leq 7$ then the boundary of the convex hull of a generic compact $k$-dimensional manifold in $n$-dimensional affine space consists of support $A_{l_{1}} \ldots A_{l_{m}}$-simplexes where $l_{1}+\ldots+l_{m} \leq n$. In particular, in the case $n=4$ it consists of support $A_{1}$-points, support $2 A_{1}$-segments, support $3 A_{1}$-triangles, support $4 A_{1}$-tetrahedrons, support $A_{3}$-points, and support $A_{1} A_{3}$-segments.

2.1. Simplest singularity $\mathcal{R}_{1}$. A germ of a convex hull in four-dimensional space has the singularity $\mathcal{R}_{1}$ if it is diffeomorphic to the germ (at the origin) of the set

$$
\mathcal{R}_{1}=\left\{(x, y, z, t) \in \mathbb{R}^{3} \times \mathbb{R}: \min _{p \geq 0}\left(\frac{p^{2}}{2}+p x+t\right) \leq 0\right\} .
$$

Remark 3 . The set $\mathcal{R}_{1}$ is the under-graph of the function of $x, y$, and $z$ which is equal to half the square of the standard distance on the real line from the point $x$ to the ray $x \geq 0$.

2.2. Angle singularities $\mathcal{R}_{2}^{0}$ and $\mathcal{R}_{2}^{ \pm}$. A germ of a convex hull in four-dimensional space has the singularity $\mathcal{R}_{2}^{0}, \mathcal{R}_{2}^{+}$, or $\mathcal{R}_{2}^{-}$if it is diffeomorphic to the germ (at the origin) of the set $\mathcal{R}_{2}(\alpha)$ where $\alpha(z)=a+z, \alpha(z)=a+z^{2}$, or $\alpha(z)=a-z^{2}$ respectively, $|a|<1$, and

$$
\mathcal{R}_{2}(\alpha)=\left\{(x, y, z, t) \in \mathbb{R}^{3} \times \mathbb{R}: \min _{p, q \geq 0}\left(\frac{p^{2}+q^{2}}{2}+\alpha(z) p q+p x+q y+t\right) \leq 0\right\}
$$

Remark 4. The set $\mathcal{R}_{2}(\alpha)$ is the under-graph of the function of $x, y$, and $z$ which is equal to half the square of the distance from the point $(x, y)$ to the coordinate angle $\{x \geq 0, y \geq 0\}$ in the plane with the Euclidean metric

$$
d s^{2}=\frac{d x^{2}-2 \alpha(z) d x d y+d y^{2}}{1-\alpha^{2}(z)} .
$$

The value of the angle is $\beta(z)=\pi-\arccos \alpha(z)$.

2.3. Singularity $\mathcal{R}_{3}$. A germ of a convex hull in four-dimensional space has the singularity $\mathcal{R}_{3}$ if it is diffeomorphic to the germ (at the origin) of the set

$$
\mathcal{R}_{3}(F)=\left\{(x, y, z, t) \in \mathbb{R}^{3} \times \mathbb{R}: \min _{p, q, r \geq 0} F(p, q, r ; x, y, z, t) \leq 0\right\}
$$

where $F$ is a polynomial whose quasihomogeneous expansion has the form $F=F_{2}+$ $F_{3}+\ldots$ if $\operatorname{deg} p=\operatorname{deg} q=\operatorname{deg} r=1, \operatorname{deg} x=\operatorname{deg} y=\operatorname{deg} z=1$, and $\operatorname{deg} t=2$,

$$
F_{2}(p, q, r ; x, y, z, t)=\frac{p^{2}+q^{2}+r^{2}}{2}+a p q+b p r+c q r+p x+q y+r z+t,
$$

and the quadratic form $\left(p^{2}+q^{2}+r^{2}\right) / 2+a p q+b p r+c q r$ is positive definite.

For example, the set $\mathcal{R}_{3}\left(F_{2}\right)$ is the under-graph of half the square of the distance from the point $(x, y, z)$ to the coordinate angle $\{x \geq 0, y \geq 0, z \geq 0\}$ in space with the Euclidean metric defined by the matrix

$$
\left\|\begin{array}{lll}
1 & a & b \\
a & 1 & c \\
b & c & 1
\end{array}\right\|^{-1} .
$$


Remark 5 . The numbers $a, b$, and $c$ are moduli of the singularity $\mathcal{R}_{3}$ with respect to diffeomorphisms. These moduli are described in [4]. Moreover, Theorem 4 implies that six moduli are among the coefficients of the quasihomogeneous component $F_{3}$ of the polynomial $F$.

2.4. Singularity $\mathcal{V}_{3}$. A germ of a convex hull in four-dimensional space has the singularity $\mathcal{V}_{3}$ if it is diffeomorphic to the germ (at the origin) of the under-graph $\mathcal{V}_{3}$ of half the square of the standard distance from the point $(x, y, z)$ to the body

$$
V_{3}=\left\{(x, y, z) \in \mathbb{R}^{3}: \forall \tau \in \mathbb{R} \tau^{4}+x \tau^{2}+y \tau+z \geq 0\right\}
$$

which is bounded by the cut swallowtail and consists of the non-negative polynomials of degree four.

2.5. Adjacencies of singularities. The singularities of convex hulls adjoin each other in the following way:

$$
\mathcal{R}_{0} \leftarrow \mathcal{R}_{1} \leftarrow \begin{gathered}
\mathcal{R}_{2}^{ \pm} \\
\downarrow \\
\mathcal{R}_{2}^{0} \\
\uparrow \\
\mathcal{V}_{3}
\end{gathered}
$$

where $\mathcal{R}_{0}$ denotes the germ of a closed half-space at a point of its boundary.

2.6. The following theorem is proved in [5].

THEOREM 1. The convex hull of a generic compact hypersurface lying in four-dimensional affine space has the following singularities:

$\mathcal{R}_{0}$ at the support $A_{1}$-points and at the interior points of the support simplexes of the $2 A_{1^{-}}, 3 A_{1-}$, and $4 A_{1}$-planes;

$\mathcal{R}_{1}$ at the endpoints of the support $2 A_{1}$-segments, at the interior points of the sides of the support $3 A_{1}$-triangles, and at the interior points of the faces of the support $4 A_{1}$ tetrahedrons;

$\mathcal{R}_{2}^{0}$ at typical vertices of the support $3 A_{1}$-triangles and at typical interior points of the edges of the support $4 A_{1}$-tetrahedrons;

$\mathcal{R}_{2}^{ \pm}$at the remaining finite number of vertices of the support $3 A_{1}$-triangles and at the remaining finite number of interior points of the edges of the support $4 A_{1}$-tetrahedrons.

2.7. Main Theorem 2 of the present paper completes this classification.

THEOREM 2. The convex hull of a generic compact hypersurface lying in four-dimensional affine space has the following singularities:

$\mathcal{R}_{3}$ at the vertices of the support $4 A_{1}$-tetrahedrons;

$\mathcal{R}_{1}$ at the interior points of the support $A_{1} A_{3}$-segments;

$\mathcal{R}_{2}^{0}$ at the points of tangency $A_{3}$ of the support $A_{1} A_{3}$-planes;

$\mathcal{V}_{3}$ at the points of tangency $A_{1}$ of the support $A_{1} A_{3}$-planes;

and the quasidegree of the polynomial $F$ from the definition of the singularity $\mathcal{R}_{3}$ is bounded by a number $d \geq 3$ which does not depend on the original hypersurface.

Theorems 1, 2 follow from Theorems 3, 4 formulated in Section 3 and proved in [1]. 


\section{Reducing to normal forms}

3.1. Normal forms of Legendre varieties. Let $B$ be a manifold and $\xi \in B$ be any of its points. A cooriented contact element to $B$ is any closed linear half-space in the tangent space $T_{\xi} B$. The point $\xi$ is called the point of applying of the contact element.

It is well known that in the space $S T^{*} B$ of all cooriented contact elements to $B$ there is a natural contact structure (hyperplane distribution satisfying the condition of maximal non-integrability): a contact element is allowed to move so that its boundary contains the velocity of its point of applying. Varieties which are tangent to the distribution and whose dimension is at most $(\operatorname{dim} B-1)$ are called Legendre.

The hyperplanes of the contact structure in the space $S T^{*} B$ are naturally cooriented outward: a contact element move in positive direction if it does not contain the velocity of the point of applying.

Later on we realize $n$-dimensional affine space as an open half-sphere in $(n+1)$ dimensional Euclidean space and work with the whole sphere which has a natural projective structure: subspaces passing through the center cut out planes of various dimensions in the sphere. Each hyperplane divides the sphere into two half-spheres, and the above definitions of a convex hull and a support hyperplane are suitable for their subsets.

A cooriented contact element to affine space or sphere is naturally identified with the pair consisting of the closed half-space and the point of applying which lies in the boundary of the half-space. Two cooriented contact elements are called complementary to each other if they consist of different closed half-spaces having common boundary and the same point of applying.

Definition 4. A cooriented contact element is called a support element to a subset $C$ of an affine space or sphere if it consists of a closed half-space containing $C$ and a point from $C$. (The point lies on the boundary of the half-space.) A cooriented contact element which is complementary to a support element is called an antisupport element. All support elements to $C$ form a subset $C^{\perp}$, all antisupport elements to $C$ form a subset $C^{\top}$.

Definition 5. A cooriented contact element is called an infinitesimal support element to a subset $C$ of a manifold if it is applied at a point $\xi \in C$ and contains the cone which is tangent to $C$ at the point $\xi$. A cooriented contact element which is complementary to an infinitesimal support element is called an infinitesimal antisupport element. All infinitesimal antisupport elements to $C$ form a subset $\widetilde{C}$.

Remark 6. The sets of all infinitesimal support and antisupport elements (to a subset of a manifold) are functorial with respect to diffeomorphisms of the manifold. In general, this is not true for the sets of support and antisupport elements.

Rem ark 7. Let $\xi \in C \subset B$ be a point of a subset $C$ of a manifold $B$. Let us consider any Riemannian metric on $B$ and some curve beginning at the point $\xi$ and possessing the following property: the distance from a point of the curve to the subset $C$ is an infinitesimal whose degree is more than one if the point approaches $\xi$. The cone lying in the tangent space $T_{\xi} B$ and consisting of the rays which are tangent to all such curves is called tangent to the subset $C$ at the point $\xi$. 
Let $R_{0}$ denote the hyperplane $s=0$ in the affine space $\mathbb{R}^{3} \times \mathbb{R}$ with coordinates $(u, v, w, s)$. Let us consider the following subsets of $R_{0}$ :

1) the hyperplane $R_{0}$ itself;

2) the half-space $R_{1}=\left\{(u, v, w) \in R_{0}: u \geq 0\right\}$;

3) the dihedral angle $R_{2}=\left\{(u, v, w) \in R_{0}: u \geq 0, v \geq 0\right\}$;

4) the octant $R_{3}=\left\{(u, v, w) \in R_{0}: u \geq 0, v \geq 0, w \geq 0\right\}$

$5)$ the body $V_{3}=\left\{(u, v, w) \in R_{0}: \forall \tau \in \mathbb{R} \tau^{4}+u \tau^{2}+v \tau+w \geq 0\right\}$ bounded by the cut swallowtail and consisting of the non-negative polynomials of degree four.

Let $(p, q, r ; u, v, w, s)$ be local coordinates on $S T^{*}\left(\mathbb{R}^{3} \times \mathbb{R}\right)$ such that a cooriented contact element applied at the point $(u, v, w, s) \in \mathbb{R}^{3} \times \mathbb{R}$ has the form $p d u+q d v+$ $r d w+d s \leq 0$, and let $E_{0} \subset S T^{*}\left(\mathbb{R}^{3} \times \mathbb{R}\right)$ denote the space of all such elements. Let $\widetilde{R_{0}}$, $\widetilde{R_{1}}, \widetilde{R_{2}}, \widetilde{R_{3}}$, and $\widetilde{V_{3}} \subset E_{0}$ be the Legendre varieties consisting of the cooriented contact elements which are infinitesimal antisupport elements to the subsets $R_{0}, R_{1}, R_{2}, R_{3}$, and $V_{3} \subset \mathbb{R}^{3} \times \mathbb{R}$ respectively. The variety $\widetilde{R_{0}}$ is smooth, the varieties $\widetilde{R_{1}}, \widetilde{R_{2}}, \widetilde{R_{3}}$, and $\widetilde{V_{3}}$ have singularities, for example, at the origin.

In the coordinates $(p, q, r ; u, v, w, s)$ :

$$
\begin{gathered}
\widetilde{R_{0}}=\{p=q=r=s=0\}, \\
\widetilde{R_{1}}=\{p u=q=r=s=0, p \geq 0, u \geq 0\}, \\
\widetilde{R_{2}}=\{p u=q v=r=s=0, p \geq 0, u \geq 0, q \geq 0, v \geq 0\}, \\
\widetilde{R_{3}}=\{p u=q v=r w=s=0, p \geq 0, u \geq 0, q \geq 0, v \geq 0, r \geq 0, w \geq 0\} .
\end{gathered}
$$

The Legendre variety $\widetilde{V_{3}}$ consists of the following three strata ( $\tau$ is a real parameter):

$$
\begin{gathered}
\left\{p=q=r=0, u \geq-2 \tau^{2}, v=-4 \tau^{3}-2 u \tau, w \geq 3 \tau^{4}+u \tau^{2}, s=0\right\}, \\
\left\{p=r \tau^{2}, q=r \tau, r \geq 0, u \geq-2 \tau^{2}, v=-4 \tau^{3}-2 u \tau, w=3 \tau^{4}+u \tau^{2}, s=0\right\} \\
\left\{p=r \tau^{2},|q| \leq r|\tau|, r \geq 0, u=-2 \tau^{2}, v=0, w=\tau^{4}, s=0\right\} .
\end{gathered}
$$

Therefore, the Legendre varieties $\widetilde{R_{l}}$ consist of $2^{l}$ strata. They can be extended to manifolds as well as the first and third strata of $\widetilde{V_{3}}$. The second stratum of the Legendre variety $\widetilde{V_{3}}$ can be extended to an irreducible algebraic variety. The union of the first and second strata of $\widetilde{V_{3}}$ is contact diffeomorphic to the Legendre variety

$$
\widetilde{\Phi_{3}}=\left\{\sigma=\Phi_{3}(\tau, \lambda), \Phi_{3, \tau}(\tau, \lambda)=0, \varkappa=-\Phi_{3, \lambda}(\tau, \lambda)\right\} \quad(\varkappa d \lambda+d \sigma=0)
$$

generated by the family

$$
\Phi_{3}(\tau, \lambda)=\frac{1}{2}\left(\tau^{4}+\lambda_{1} \tau^{2}+\lambda_{2} \tau+\lambda_{3}\right)^{2}
$$

The reducing contact diffeomorphism is given by the formula $(\varkappa, \lambda, \sigma)=(p, q, r ; u, v$, $\left.w-r, s+r^{2} / 2\right)$.

DeFinition 6. We say that a three-dimensional Legendre variety has the singularity $\widetilde{R_{1}}, \widetilde{R_{2}}, \widetilde{R_{3}}$, or $\widetilde{V_{3}}$ at some point if its germ at this point is (up to a local diffeomorphism respecting the contact structures and their coorientations) respectively the germ of the Legendre variety $\widetilde{R_{1}}, \widetilde{R_{2}}, \widetilde{R_{3}}$, or $\widetilde{V_{3}} \subset E_{0}$ at the origin. 
These singularities of Legendre varieties adjoin each other in the following way:

$$
\widetilde{R_{0}} \leftarrow \widetilde{R_{1}} \leftarrow \underset{\widetilde{R_{2}}}{\widetilde{\uparrow}} \leftarrow \widetilde{R_{3}}
$$

where the indices are equal to the codimensions of the strata of a Legendre variety which consist of the corresponding singular points.

TheOREm 3. Let $M \subset S^{4}$ be a compact smooth hypersurface, $[M] \subset S^{4}$ be its convex hull, $[M]^{\perp} \subset S T^{*} S^{4}$ be the set of all cooriented contact support elements to $[M]$, and $\pi: S T^{*} S^{4} \rightarrow S^{4}$ be the natural projection.

Then $[M]^{\perp}$ is a Legendre variety which is uniquely projected onto its front $\pi\left([M]^{\perp}\right)$ which is the boundary of the convex hull of the original hypersurface $M$. If the hypersurface $M$ is generic, the Legendre variety $[M]^{\perp}$ can have only the above singularities $\widetilde{R_{1}}, \widetilde{R_{2}}$, $\widetilde{R_{3}}$, and $\widetilde{V_{3}}$. Moreover:

1) the Legendre variety $[M]^{\perp}$ is smooth above the support $A_{1}$-points and above the interior points of the support simplexes of the $2 A_{1^{-}}, 3 A_{1^{-}}$, and $4 A_{1}$-planes of the hypersurface $M$;

2 ) the singularities $\widetilde{R_{1}}$ appear above the endpoints of the support $2 A_{1}$-segments, above the interior points of the sides of the support $3 A_{1}$-triangles, above the interior points of the faces of the support $4 A_{1}$-tetrahedrons, and above the interior points of the support $A_{1} A_{3}$-segments of the hypersurface $M$;

3 ) the singularities $\widetilde{R_{2}}$ appear above the vertices of the support $3 A_{1}$-triangles, above the interior points of the edges of the support $4 A_{1}$-tetrahedrons, and above the points of tangency $A_{3}$ of the support $A_{1} A_{3}$-planes of the hypersurface $M$;

4) the singularities $\widetilde{R_{3}}$ appear above the vertices of the support $4 A_{1}$-tetrahedrons of the hypersurface $M$;

$5)$ the singularities $\widetilde{V_{3}}$ appear above the points of tangency $A_{1}$ of the support $A_{1} A_{3}$ planes of the hypersurface $M$.

\subsection{Normal forms of Legendre bundles. A Legendre mapping is a diagram}

$$
L^{n-1} \hookrightarrow E^{2 n-1} \rightarrow B^{n}
$$

consisting of an embedding of a Legendre variety $L^{n-1}$ into the space $E^{2 n-1}$ with a cooriented contact structure and a Legendre bundle $E^{2 n-1} \rightarrow B^{n}$. (A smooth bundle whose fibers are Legendre manifolds is called Legendre.) Equivalence of Legendre mappings is a commutative diagram

$$
\begin{array}{lllll}
L & \hookrightarrow & E & \rightarrow & B \\
\uparrow & & \uparrow & & \uparrow \\
L^{\prime} & & & & E^{\prime}
\end{array}
$$

where the middle vertical arrow is a diffeomorphism sending the cooriented contact structures to each other.

Legendre bundles are locally given with the help of the generating families defined below. 
Let us consider again the space $E_{0}$ of cooriented contact elements $\varkappa d \lambda+d s \leq 0$ applied at the points $(\lambda, s) \in \mathbb{R}^{3} \times \mathbb{R}$ where $\varkappa=(p, q, r), \lambda=(u, v, w)$. The cooriented contact structure on $E_{0}$ is given by the zero subspaces of the form $\varkappa d \lambda+d s$.

Let $F:\left(\mathbb{R}^{3} \times \mathbb{R}^{3} \times \mathbb{R}, 0\right) \rightarrow(\mathbb{R}, 0)$ be a germ of a family of smooth functions of $\varkappa$ which depend smoothly on the parameters $\mu=(x, y, z) \in \mathbb{R}^{3}$ and $t \in \mathbb{R}$. Let $F_{\varkappa}(0)=0$ and let $F$ satisfy the condition of non-degeneracy at the origin:

$$
\operatorname{det}\left\|\begin{array}{cc}
F_{\varkappa \mu} & F_{\varkappa t} \\
F_{\mu} & F_{t}
\end{array}\right\| \neq 0
$$

Then $F$ is called the generating family of the germ

$$
\pi:\left(E_{0}, 0\right) \rightarrow\left(\mathbb{R}^{3} \times \mathbb{R}, 0\right), \quad \pi(\varkappa, \lambda, s)=(\mu, t)
$$

of the Legendre bundle whose fibers are given by the formula

$$
\pi^{-1}(\mu, t)=\left\{(\varkappa, \lambda, s) \in E_{0}: \lambda=F_{\varkappa}(\varkappa, \mu, t), s=F(\varkappa, \mu, t)-\varkappa \lambda\right\} .
$$

This bundle is correctly defined in a neighborhood of the origin in view of the nondegeneracy of $F$.

Thus $\varkappa$ are local coordinates on the fibers of the germ $\pi,(\mu, t)$ are local coordinates on its base, and the cooriented contact structure on $E_{0}$ is defined by the form $F_{\mu} d \mu+F_{t} d t$.

For example, the natural Legendre bundle $(\varkappa, \lambda, s) \mapsto(\lambda, s)$ is given by the generating family $\varkappa \mu+t=p x+q y+r z+t$.

THEOREM 4. Let $L \subset S T^{*} S^{4}$ be a Legendre variety with the singularities $\widetilde{R_{1}}, \widetilde{R_{2}}$, $\widetilde{R_{3}}$, or $\widetilde{V_{3}}$ and $\pi: S T^{*} S^{4} \rightarrow S^{4}$ be a generic Legendre bundle. If $\pi(L)$ is a continuously differentiable manifold then the germ of the Legendre mapping $L \hookrightarrow S T^{*} S^{4} \stackrel{\pi}{\longrightarrow} S^{4}$

1) at each smooth point of the variety $L$ is equivalent to the germ $\left(\widetilde{R_{0}}, 0\right) \hookrightarrow\left(E_{0}, 0\right) \rightarrow$ $\left(\mathbb{R}^{3} \times \mathbb{R}, 0\right)$ of a Legendre mapping such that its second arrow is given by the generating family

$$
p x+q y+r z+t
$$

2) at each singular point $\widetilde{R_{1}}$ of the variety $L$ is equivalent to the germ $\left(\widetilde{R_{1}}, 0\right) \hookrightarrow$ $\left(E_{0}, 0\right) \rightarrow\left(\mathbb{R}^{3} \times \mathbb{R}, 0\right)$ of a Legendre mapping such that its second arrow is given by the generating family

$$
\frac{p^{2}}{2}+p x+q y+r z+t
$$

3) at each singular point $\widetilde{R_{2}}$ of the variety $L$ is equivalent to the germ $\left(\widetilde{R_{2}}, 0\right) \hookrightarrow$ $\left(E_{0}, 0\right) \rightarrow\left(\mathbb{R}^{3} \times \mathbb{R}, 0\right)$ of a Legendre mapping such that its second arrow is given by one of the generating families having the form

$$
\frac{p^{2}+q^{2}}{2}+\alpha(z) p q+p x+q y+r z+t
$$

where $\alpha(z)=a+z$ for a typical singular point $\widetilde{R_{2}}, \alpha(z)=a \pm z^{2}$ for the remaining finite number of singular points $\widetilde{R}_{2}$, and $|a|<1$ is a continuous invariant;

4) at each singular point $\widetilde{R_{3}}$ of the variety $L$ is equivalent to the germ $\left(\widetilde{R_{3}}, 0\right) \hookrightarrow$ $\left(E_{0}, 0\right) \rightarrow\left(\mathbb{R}^{3} \times \mathbb{R}, 0\right)$ of a Legendre mapping such that its second arrow is given by a polynomial generating family $F(p, q, r ; x, y, z, t)$ whose quasihomogeneous expansion has 
the form $F=F_{2}+\ldots+F_{d}$ if $\operatorname{deg} p=\operatorname{deg} q=\operatorname{deg} r=1, \operatorname{deg} x=\operatorname{deg} y=\operatorname{deg} z=1$, and $\operatorname{deg} t=2$, where

$$
F_{2}=\frac{p^{2}+q^{2}+r^{2}}{2}+a p q+b p r+c q r+p x+q y+r z+t,
$$

$a, b$, and $c$ are continuous invariants, the quadratic form $\left(p^{2}+q^{2}+r^{2}\right) / 2+a p q+b p r+c q r$ is positive definite, among the coefficients of $F_{3}$ there are six continuous invariants, and the number $d \geq 3$ depends on neither $L$ nor $\pi$;

5) at each singular point $\widetilde{V_{3}}$ of the variety $L$ is equivalent to the germ $\left(\widetilde{V_{3}}, 0\right) \hookrightarrow$ $\left(E_{0}, 0\right) \rightarrow\left(\mathbb{R}^{3} \times \mathbb{R}, 0\right)$ of a Legendre mapping such that its second arrow is given by the generating family

$$
\frac{p^{2}+q^{2}+r^{2}}{2}+p x+q y+r z+t .
$$

Remark 8. In the case 5) the generating family can be reduced to the simpler form

$$
\frac{q^{2}+r^{2}}{2}+p x+q y+r z+t .
$$

\section{References}

[1] I. A. Bogaevski, Singularities of convex hulls of three-dimensional hypersurfaces, Trudy Mat. Inst. Steklov. 221 (1998), 81-100 (in Russian); English transl.: Proc. Steklov Inst. Math. 221 (1998), 71-90.

[2] A. B. Givental', Singular Lagrangian manifolds and their Lagrangian mappings, Itogi Nauki i Tekhniki, Akad. Nauk SSSR, Ser. Sovremennye Problemy Matematiki 33, VINITI, Moscow, 1988, 55-112 (in Russian); English transl.: J. Soviet Math. 52 (1990), 3246-3278.

[3] V. D. Sedykh, Functional moduli of singularities of convex hulls of manifolds of codimension 1 and 2, Mat. Sb. (N.S.) 119 (1982), 233-247 (in Russian); English transl.: Math. USSR-Sb. 47 (1984), 223-236.

[4] V. D. Sedykh, Singularities of convex hulls, Sibirsk. Mat. Zh. 24 (1983), no. 3, 158-175 (in Russian); English transl.: Siberian Math. J. 24 (1983), 447-461.

[5] V. D. Sedykh, Stabilization of the singularities of convex hulls, Mat. Sb. (N.S.) 135 (1988), 514-519 (in Russian); English transl.: Math. USSR-Sb. 63 (1989), 499-505.

[6] V.D.Sedykh, The sewing of the swallowtail and the Whitney umbrella in a four-dimensional control system, Trudy GANG im. I. M. Gubkina, Oil and Gas, Moscow, 1997, 58-68 (in Russian).

[7] V. M. Zakalyukin, Singularities of convex hulls of smooth manifolds, Funktsional. Anal. i Prilozhen. 11 (1977), no. 3, 76-77 (in Russian); English transl.: Functional Anal. Appl. 11 (1978), 225-227.

[8] V. M. Zakalyukin, R. M. Roberts, Stability of Lagrangian manifolds with singularities (Russian), Funktsional. Anal. i Prilozhen. 26 (1992), no. 3, 28-34; English transl.: Funct. Anal. Appl. 26 (1992), 174-178. 